\title{
Complex networks and computing
}

\author{
Shuigeng ZHOU ${ }^{\dagger}$, Zhongzhi ZHANG \\ Shanghai Key Lab of Intelligent Information Processing, School of Computer Science, Fudan University, Shanghai 200433, China
}

(c) Higher Education Press and Springer-Verlag 2009

Nowadays complex networks are pervasive in various areas of science and technology. Popular examples of complex networks include the Internet, social networks of collaboration, citations and co-authoring, as well as biological networks such as gene and protein interactions and others. Complex networks research spans across mathematics, computer science, engineering, biology and the social sciences. Even in computer science area, increasing problems are either found to be related to complex networks or studied from the perspective of complex networks, such as searching on Web and P2P networks, routing in sensor networks, language processing, software engineering etc. The interaction and mergence of complex networks and computing is inspiring new chances and challenges in computer science.

In this special section, we aim to offer readers the state of the art in the interdisciplinary field of complex networks and computing. The articles in this section cover a wide array of issues on topics that fall into two tracks: complex network computing and computing based on complex networks, each of which contains seven carefully selected papers. Papers of the first track cover mainly the issues of complex network modeling and statistical computation, and papers in the second track deal with various computing issues from the perspective of complex networks or by the philosophy of complex networks.

In the track of complex network computing, Zonghua Liu et al. present a novel approach to study how the topological structure of a complex network influences the concrete process of epidemic spreading. Shengqi Yang et al. present a comprehensive study on a massive anonymous call records obtained from a major mobile service operator by multidimensional analysis. Bin Wu et al. investigate users' structural properties in mobile call networks from a novel perspective, structure correlation between connected users. Lili Rong et al. consider the mechanisms of real networks and define a cost function to describe different hierarchies of networks to measure nodes' importance. Chengyi Xia et al. present a new epidemic SIS (Susceptible-Infected-Susceptible) model to investigate the spreading behavior on networks with dynamical topology and community structure. Ji Liu et al. present a new method of detecting community in networks based on representative energy. Huan Li introduces two scale-free network models of accelerating growth with undirected and directed links and obtains their predicted scale-free exponents by theoretic analysis.

In the track of computing based on complex networks, Quanqing Xu et al. propose an automatic and economical hybrid information retrieval approach based on cooperative cache. Detailed mathematical analysis on the cache hit ratio and simulation experiments in NS-2 are also presented. Weifeng Pan et al. propose an approach to refactor the class structures of $\mathrm{OO}$ (Object-Oriented) software systems based on software network structure analysis. Penggang Sun et al. propose a fast iterative-clique percolation method (ICPM) for identifying overlapping functional modules in PPI (protein-protein interaction) networks. Jialu Hu et al. present a methodology to evaluate the performance of three algorithms, ESA, ESU and RAND- ESU. Qiang Guo et al. present an improved

†Prof. Shuigeng ZHOU and Dr. Zhongzhi ZHANG are the guest editors of the special section on Complex Network and Computing

E-mail:\{sgzhou, zhangzz\}@fudan.edu.cn 
collaborative filtering (MCF) algorithm by using the heat diffusion process to generate the user correlation. This algorithm has remarkably higher accuracy than the standard collaborative filtering (CF) using Pearson correlation. Shiwen SUN et al. give an empirical study on header file collaboration networks (HFCNs) where each node represents a header file and two nodes are connected if corresponding header files are both included in the same source file at least once. Xuejun Liu et al. introduce a new routing protocol based on the concept of small worlds where the addition of a small number of long-range links in highly clustered networks results in significant reduction in the average path length.

Many people have contributed to this special section. We are grateful to all authors contributing to the special section. We appreciate the reviewers who helped in reviewing the submissions and selecting quality papers. We thank Prof. Ruqian Lu, the Executive Editor-in-Chief of Frontiers of Computer Science in China for supporting this special section. Especially we thank Dr. Wei Cheng for her impressive patience, coordination and cooperation in preparation the special section. We hope this special section is interesting and helpful to the readers.

June 28, 2009 